\title{
Promoting Creativity at Work - Implications for Scientific Creativity
}

\author{
SANDRA OHLY \\ University of Kassel, Pfannkuchstr. 1, D-34121 Kassel, Germany. \\ Email: ohly@uni-kassel.de
}

\begin{abstract}
Creativity is the generation of novel and useful ideas. Organizations, including universities and other research institutions, need to develop novel and useful products to satisfy constantly evolving customer needs. Furthermore, organizational procedures and processes develop over time, and continuous improvements in processes contribute to organizational efficiency. Thus, the development of novel and useful ideas in relation to products, procedures and services is mandated from many employees in modern organizations, including researchers and scientists. This creativity can take on many different forms, for example unconventional solutions to a wide variety of problems related to research, teaching and administration. Because many of these problems are comparable to that of workers in all kinds of jobs, I believe it is possible to draw on the rich knowledge in the field of organizational creativity to provide some insights into how to foster scientific creativity. The work of researchers and scientists is in many ways comparable to that of knowledge workers in other kinds of organizations: they are high-level employees who apply knowledge to do their work, oftentimes using creative thinking. A rich body of literature has dealt with creativity in these jobs where creativity is a core requirement, and has examined the organizational factors related to creativity. Before I summarize this research and highlight some important research findings concerning the rewards for creativity, the design of jobs, the processes leading to creativity, the organizational climate, and daily work events associated with creativity, I will disentangle different forms of creativity at work and describe a general model of creativity in the context of work.
\end{abstract}

\section{Types of Creativity}

Creativity is not only a characteristic of exceptional individuals, such as Nobel Prize winners or of certain jobs such as artists, for whom novelty and originality is the defining feature, but also for individuals in other kinds of jobs. There are individuals who regularly engage in creative work such as producing new solutions or designs that are widely useful. In these jobs, recognizing problems is part of the core work 
task. ${ }^{1}$ Unsworth coined the concept of creative requirements. Consider for example a marketing professional who is required to develop a new campaign for a customer and needs to find a specific problem in previous campaigns in order to satisfy customer needs. Here, a creative product is expected, and the marketing professional's performance is evaluated according to whether or not he or she delivers this product. The importance of creativity is, however, not limited to these jobs. In other jobs that are not immediately associated with creativity, problems are discovered on one's own initiative; for example, if a nurse realizes that the scheduling of work needs to be improved to better meet the patients' needs. In these types of jobs, creativity can be considered as extra-role performance as creativity is not an explicit component of the job description. Most research on creativity has examined the processes and circumstances leading to creativity in areas where creative requirements are high. As described above, these jobs are similar to the ones of researchers and scientists. However, it is an open question if all these jobs have high levels of creative requirements. Below, I will refer to this concept again when describing research findings.

Creativity also differs in the extent to which a problem is clearly stated for the person to work on. Some types of creativity rely on individuals first finding a problem to work on, and defining it in the first place. This distinction is important in scientific creativity as well: does a research project respond to a call for more research on a specific topic, or does it aim at finding a completely new approach to a topic? How much autonomy is there to decide what topic to study? Although this distinction is important, so far little research has specifically examined the processes leading to creativity in open versus closed problems.

Creativity can be regarded as a process consisting of different steps: first, a problem needs to be recognized or presented to be solved. ${ }^{2}$ In the second step, the preparation phase, information on the problem space is collected from external sources (written material, colleagues, friends) or recollected from memory. In the third step, the idea generation phase, multiple solutions to the problems are generated. These potential solutions are then evaluated in the fourth step: which solution best solves the problem and meets all requirements? To become effective, the solution needs to be implemented in the fifth and final step. Individuals who are motivated are thought to engage in the problem finding process more often, and exert more effort in the idea generation phase. This is why research in organizational creativity often focuses on motivating factors. In addition, individuals having a good knowledge of their field are thought to be better at identifying relevant information and at judging what solutions fit best. Knowledge can be acquired in formal or informal ways, in education settings or by learning on the job. Finally, individuals need specific creativity-related skills such as divergent thinking abilities, and an innovative cognitive style or a disposition to question traditional approaches. These creativity relevant skills will provide the necessary input for generating solutions to problems.

Research on creativity has examined personal and contextual factors that influence creativity, including personality, abilities, self-regulatory processes, and job design characteristics. A long debate has focused on the detrimental effects of rewards on creativity. I will provide an overview of the findings concerning this effect. 
Furthermore, in order to provide some insights into how to foster scientific creativity, I will focus on contextual characteristics, including job design, organizational climate, and processes enhancing creativity. This choice is motivated by evidence that contextual characteristics and certain processes exert a strong influence on creativity. ${ }^{3,4}$ Contextual characteristics as well as the intra-individual processes that occur during a work day are malleable and can thus be organized in a way to promote scientific creativity. In what follows, I will review research on these factors: First, I will review research on extrinsic rewards and creativity and second, the contextual characteristics and the work environment that foster creativity in organizations. Third, I will turn to research on the processes involved in creativity.

\section{Extrinsic Rewards, Intrinsic Motivation and Creativity}

Intrinsic motivation refers to motivation stemming from the task itself, whereas extrinsic motivation refers to motivation to gain some extrinsic reward. A long research tradition focuses on intrinsic motivation as a precursor of creativity, and in this tradition, rewards are believed to reduce intrinsic motivation based on experiments that paying children to do an interesting task leads to lower engagement with the task once the reward has been removed. ${ }^{5}$ There is a large disagreement about the generalizability of this effect, and research supporting the notion that rewards negatively impact intrinsic motivation in the adult populations and in the work setting is scarce.

Furthermore, rewards might even enhance creativity when given contingent on creativity performance. Contingent implies that when and only when a creative performance occurs, the reward is given. The crucial issue then becomes to identify the types of creative achievements that are worth rewarding based on reliable assessments of creativity. This assessment is challenging because creativity is often domain- and context-specific, which limits the number of individuals able to make this assessment. Furthermore, the usefulness of an idea will only be evident after some time when it becomes clear that a problem is solved in a better way than was previously possible.

Both intrinsic and extrinsic motivation might enhance creativity because individuals are more motivated to engage in the processes leading to creativity, and to show persistence when developing ideas. Extrinsic rewards might indicate which kind of behaviour is desired in organizations. In other words, extrinsic rewards that are given contingent on creative performance might foster creativity by enhancing creative requirements. Interestingly, there is evidence that extrinsic rewards might not be necessary or even detrimental when individuals work in jobs high in creative requirements, and when they have an innovative thinking style. ${ }^{6}$

I will now turn to job design factors that have been linked to creativity, oftentimes with a motivational perspective.

\section{Considering the Context: Job Design and Social Context}

Creativity requirements are high in jobs that are characterized by high levels of autonomy, high demands, and high levels of complexity. ${ }^{7}$ Autonomy refers to the freedom of an individual in how to carry out their job (also called job control). 
Demands refer to high standards and requirements to work hard. Job complexity refers to the number of elements that need to be considered in doing the job. A high level of job complexity implies that there is a high variation in the core job tasks, and that a variety of skills are needed to do the job. Researchers' and scientists' jobs are prototypical of these jobs.

Research has linked autonomy and job complexity not only to creativity requirements but also to higher creative performance. Various processes have been outlined that explain how these job characteristics contribute to creativity: individuals in complex jobs with high levels of autonomy have many options in how to carry out their tasks so that experimentation in working methods is possible, which leads to creativity. Furthermore, both autonomy and job complexity are intrinsically motivating, and intrinsic motivation is linked to creativity. In addition, individuals in complex, autonomous jobs experience higher levels of positive affect, which facilitates creative problem-solving.

In contrast to autonomy and job complexity, the relationship between demands and creativity is more complex, and research has yielded mixed results, including positive, negative, and curvilinear (inverted U-shaped relationships). The overall mixed pattern of findings has led researchers to the distinction between challenging work (having a sense of important work to do) and workload pressure (excessively high demands). Whereas challenging work seems to stimulate creativity, workload pressure is seen as an obstacle that hinders creative thinking. In fact, time pressure can be appraised as challenging, and this appraisal is linked to daily creativity. ${ }^{8}$ However, the question remains, under which conditions are high demands perceived as challenging and when are they perceived as hindrances for creativity? This perception might depend on the level of internal and external resources available to cope with these demands. It might be that individuals high on self-efficacy (the belief that one is able to execute the required course of action to achieve a goal) see high demands as challenges, whereas individuals low on self-efficacy see them as workload pressures. Furthermore, people face qualitatively different demands at work. Demands stemming from tight deadlines on important projects may lead to the perception of challenges, whereas demands stemming from unnecessary work tasks (bureaucratic work) are associated with less favourable perceptions and reactions. In order to foster creative outputs in research, unnecessary tasks will thus need to be reduced or eliminated.

Support from colleagues and supervisors can take on two different forms: informational support or emotional support. ${ }^{9}$ Informational support indicates that valuable information is exchanged. Emotional support indicates that individuals care about each other and provide encouragement. Both forms of support, but primarily informational support, are provided when work groups are characterized by high levels of communication.

Work groups that communicate about potential work problems, openly exchange knowledge and ideas, and discuss solutions to problems are expected to display higher levels of creativity than work groups where communication is lacking or superficial. Group communication is essential when difficult, non-standardized tasks 
have to be completed, or when creative requirements are high. Communication in the work group has been linked to both individual and team innovation in a recent metaanalysis. ${ }^{4}$ However, not all communication provides novel insights or stimulates new thoughts. Only when diverse opinions are expressed is there a potential for creativity.

Emotional support is high in work groups in which members like, support, and trust each other. When individuals feel safe to express their ideas and explore new procedures, they are more likely to come up with novel ideas. However, emotional support also entails the risk that individuals are primarily motivated to maintain the positive affective tone and 'shy away from criticizing each other's ideas' (Ref. 4, p. 139). To enhance creativity it is essential that problems are discussed openly and in a constructive way.

\section{Processes Linking Job Context and Creativity}

Previous research has largely rested on the assumption that certain characteristics, such as autonomy, job complexity and support from others, promote intrinsic motivation and thereby enhance creativity. The basic rationale was that individuals in jobs characterized by autonomy, job complexity and support will be more creative because they are intrinsically motivated to work on problems and, therefore, persist longer. While some research has supported this idea, evidence is equivocal. In my own research, I explored additional mechanisms for how creativity can be fostered in these jobs. To this aim, I studied the creativity of 177 employees from diverse jobs and from various organizations in production and insurance. Employees were asked to rate their own level of creativity using a well-established measure consisting of statements such as 'I often generate novel and useful ideas'. Because employees might tend to overrate their level of creativity, I also aimed at assessing their supervisors' view using the same statements but targeting the focal employee.

In addition to intrinsic motivation, I also assessed trust in the supervisor and creative requirements. Trust in the supervisor is the willingness to be vulnerable to the action of the supervisor. It involves the willingness to take risks. Developing ideas and discussing them with a supervisor who might disagree involves a certain level of riskiness. Therefore, I expected that individuals will be more creative when they feel supported and trust their supervisor as a consequence. I also expected, based on previous research, that individuals perceiving their job as high in autonomy, complexity and time pressure would feel higher creative requirements, and become more creative as a result. The relationships between job design, creative requirements, trust, and creativity partially supported my hypotheses. Creative requirement indeed explained the effect of job autonomy on creativity. Furthermore, trust was responsible for the relationship between supervisor support and creativity. Interestingly, although intrinsic motivation predicted creativity over and above the level of trust and creative requirements, it was not the link between job design and creativity. These results were virtually identical when using supervisor ratings as an outcome instead of employees' ratings of creativity. These results suggest that there are important mechanisms linking job design and creativity that have been neglected in previous 
theorizing and research, namely creative requirements and trust. It is also possible that there are additional mechanisms. For example, experimental research in the laboratory using creativity tasks indicated that individuals become more creative when in a good mood, when they feel elated and enthusiastic. Below, I will elaborate on how this research can inform organizational practices in fostering creativity.

\section{Macro Perspective: Organizational Culture and Climate}

Many different approaches for studying organizational culture and climate exist. A useful clustering of different approaches describes three different facets of organizational climate/culture that can be used to organize research findings: the affective facet, the cognitive facet, and the instrumental facet. ${ }^{10}$ The affective facet encompasses the perception of employees that there are positive social relationships in the organization, and that individuals, including supervisors, care about each other and treat each other with respect. This facet is similar to perceived support from supervisors and colleagues, but targets the relationships in the whole organization. The cognitive facet encompasses the perception that learning and improvement are important, that change is accepted and innovation is supported. Furthermore, individuals perceive that it is okay to make independent, autonomous decisions, and organizations use formal recognition and awards based on ability and effort. The instrumental facet encompasses the perception that structure and hierarchy are important in the organization, that extrinsic rewards of pay are used, and that there are high levels of demands and challenges. While there is clear evidence for the instrumental facet, the affective and cognitive facets are linked to higher levels of employee creativity. ${ }^{3}$ Furthermore, there is a strong resemblance to research on job design and social support. Thus, it seems likely that the underlying mechanisms are similar: in an organization characterized by an affective climate, employees will trust each other and share ideas openly without fear that negative consequences will follow. Furthermore, in an organization characterized by a cognitive climate, employees will see a high necessity to develop novel and useful ideas (creative requirements). It is important to note that both climate facets can coexist so that organizations can be characterized by high levels of both facets at the same time. Not only is creativity a likely consequence, but employees are also more satisfied and more committed to their organizations. ${ }^{10}$

\section{Micro Perspective: Affective Work Events}

As mentioned above, research shows that individuals can be more creative when experiencing positive affect (enthusiastic, elated), not only in laboratory studies. A study examining the creative process engagement of $\mathrm{PhD}$ and other research students suggests that they engage in more creative processes (problem identification, information processing, and idea/alternative generation) on days when the level of positive affect was high compared to when it was low. This effect was especially pronounced when the students exhibited a high learning orientation. For these 
students, experiencing positive affect indicates that they are making good task progress, and they are more motivated to engage in creative processes as a result.

Our own research suggests that goal progress or task mastery is an important precursor of positive affect at work. ${ }^{11}$ Based on more than 500 daily diary entries of 218 employees, we were able to identify four different types of positive events that occur at work. Among them, the experiencing of goal progress, task accomplishment, or problem-solving was most frequent and also most strongly related to positive affect. Interestingly, the experience of appreciation or positive feedback, although widely believed to be important for employees, was less frequent, and also less strongly linked to positive affect. Taking these findings together, research on the micro-level, studying the daily experiences of employees at work, suggests that positive affect arises when individuals are able to do their job, and that this positive affect fosters their daily engagement in creative processes. It follows, because creative process engagement can be linked to creative outputs, that individuals get more creative when experiencing these kinds of positive events at work.

\section{Implications for Fostering Scientific Creativity and for University Governance}

Recently, Trakman ${ }^{12}$ described different models of university governance and discussed their advantages and disadvantages: faculty, corporate, trustee governance, stake holders, and amalgam models of governance. The research on contextual influences on creativity suggests additional arguments, pro and con some of these university governance models.

Based on the research presented above, one can conclude that organizations, including universities and other research institutions, need to build trusting relationships among their employees and foster a supportive climate. This could be achieved by having employees participate in decision making, by training supervisors to show appreciation, or by establishing norms that everyone needs to be treated with dignity and respect. As far as a trustee governance structure supports trust among organizational members, one can expect creativity to benefit.

Supervisors have a prominent role in these kinds of initiatives, because they act as role models for others, and their behaviour indicates what is normal in an organization. In this regard, there is a certain danger in competition for reputation, research money and publications. When individuals compete, they will be less likely to share ideas openly and support each other. Thus, initiatives based on competition such as the excellence initiative might have tangible side effects that need to be taken into account.

A second implications is that organizations need to strengthen the perception that creativity is required. In corporate governance structures, key performance indicators are used to manage and monitor the organization's success. To strengthen the perception that creativity is required, these key performance indicators need to include indicators of creative performance. A performance appraisal system that is based on quantifiable indicators of performance, such as number of articles published, amount of grant money received, or number of $\mathrm{PhD}$ students who graduated, 
tends to neglect the question of novelty and usefulness, or relies on less than perfect indicators, e.g. the reputation of a journal to judge the quality of a publication. A more rigorous assessment of the novelty and usefulness of ideas (i.e. quality) applied in an organization would strengthen the perception of creative requirements so that researchers and scientists perceive it is not only important to produce quantifiable output.

The third implication is for universities and other research institutions to provide a working environment that allows employees to experience positive affect, making it more likely that they experience the kind of affective events linked to positive affect, most notably goal attainment, problem solving or task-related success. This could be achieved by providing the necessary resources so that employees can make good progress in their primary task (research), and reduce or eliminate secondary tasks that hinder goal progress by diverting attention. The faculty governance model has been criticized because academic staff often lack the skill or interest in activities involved in governing the university, which might undermine the organization's success. Research on organizational creativity also suggests detrimental outcomes of this governance structure for individuals' creativity: when they lack interest and skills in the required activities, it is unlikely that they will experience positive affect. Furthermore, these activities draw on an important resource: working time.

Taken together, research on organizational creativity provides some insights on how to foster scientific creativity. Aside from using monetary rewards, fostering positive affect, increasing social support and creating an organizational climate conducive to creativity, and explicitly recognizing creative achievements seem to be promising. This research provides arguments for and against different university governance models.

\section{References}

1. K.L. Unsworth (2001) Unpacking creativity. Academy of Management Review, 26, pp. 289-297.

2. T.M. Amabile (1996) Creativity in the Context (Boulder, CO: Westview Press)

3. M.M. Hammond, N.L. Neff, J.L. Farr, A.R. Schwall and X. Zhao (2011) Predictors of individual-level innovation at work: A meta-analysis. Psychology of Aesthetics, Creativity, and the Arts, 5, pp. 90-105.

4. U.R. Hülsheger, N. Anderson and J.F. Salgado (2009) Team-level predictors of innovation at work: a comprehensive meta-analysis spanning three decades of research. Journal of Applied Psychology, 94, pp. 1128-1145.

5. B.A. Hennessey and T.M. Amabile (2010) Creativity. Annual Review of Psychology, 61, pp. 569-598.

6. M. Baer, G.R. Oldham and A. Cummings (2003) Rewarding creativity: When does it really matter? The Leadership Quarterly, 14(4), pp. 569-586.

7. S. Ohly and R. Bledow (2014) Performance and well-being in creative work. In M.v. Veldhoven and R. Peccei, (Eds.) Well-being and Performance at Work: The Role of Context (London: Psychology Press), pp. 75-91.

8. S. Ohly and C. Fritz (2010) Work characteristics, challenge appraisal, creativity, and proactive behavior: A multi-level study. Journal of Organizational Behavior, 31, pp. 543-565. 
9. N. Madjar (2008) Emotional and informational support from different sources and employee creativity. Journal of Occupational \& Organizational Psychology, 81, pp. 83-100.

10. J.Z. Carr, A.M. Schmidt, J.K. Ford and R.P. DeShon (2003) Climate perceptions matter: A meta-analytic path analysis relating molar climate, cognitive and affective states, and individual level work outcomes. Journal of Applied Psychology, 88, pp. 605-619.

11. S. Ohly and A. Schmitt (2015) What makes us enthusiastic, angry, feeling at rest or worried? Development and validation of an affective work events taxonomy using concept mapping methodology. Journal of Business and Psychology, 30(1), pp. $15-35$.

12. L. Trakman (2008) Modelling university governance. Higher Education Quarterly, 62, pp. 63-83.

\begin{abstract}
About the Author
Sandra Ohly has been professor in business psychology at the University of Kassel, Germany since 2010. She received her PhD from the Technical University of Braunschweig, Germany in 2005, and her habilitation from the Goethe University Frankfurt, Germany, in 2010. Her research focuses on well-being, creativity and proactive behaviour at work. She is also interested in affective and motivational processes, oftentimes using diary methods. In a recent research project she examined how smartphone use after hours relates to well-being and work-home interference. Her research has been published in Journal of Organizational Behavior, Journal of Applied Psychology, Journal of Occupational and Organisational Psychology and Journal of Business and Psychology. She is associate editor of Journal of Personnel Psychology, and a member of the editorial board of Journal of Organizational Behavior, Journal of Occupational and Organisational Psychology and Journal of Business and Psychology.
\end{abstract}

